\title{
Penile Lesion from Gunshot Wound: a 43-Case Experience
}

\author{
Andre G. Cavalcanti, Renato Krambeck, Alexandre Araujo, Carlos H. Manes, Luciano A. \\ Favorito
}

Section of Urology, Souza Aguiar Municipal Hospital, Rio de Janeiro, RJ, Brazil

\begin{abstract}
Objective: To demonstrate the main aspects of diagnosis, treatment and follow-up of 43 patients with gunshot wounds to the penis.

Materials and Methods: The location of the lesion, the presence of associated lesions, the performance of complementary exams, surgical treatment, postoperative complications and long term follow-up of 43 patients with penile lesions from gunshot wounds were retrospectively analyzed.

Results: Of 43 cases assessed, 41 were submitted to surgical exploration (95.3\%) and 2 were submitted to conservative treatment $(4.7 \%)$. We found penile lesions involving the corpus cavernosum in 37 cases; the remaining 4 patients presented no lesions involving the corpus cavernosum, urethra or testicles but did in the superficial structures. Ten cases presented an association with testicular lesions and 14 cases association with anterior urethral lesions.

Conclusion: Penile lesions from gunshot wounds should be treated with immediate surgical intervention. In exceptional situations featuring superficial lesions only conservative treatment may be applied.
\end{abstract}

Key words: penis; urethra; wounds, gunshot; surgical procedures, operative

Int Braz J Urol. 2006; 32: 56-63

\section{INTRODUCTION}

Gunshot wounds to the genitalia are not frequent (1), however they need to be immediately investigated to assess the extension of the lesion to reproductive organs and prevent complications, such as bleeding, infection, penile curvature, erectile dysfunction and urethral stenosis (2).

The penis can be injured in an isolated way or in association with scrotal traumatism (3). Even though they are not frequent, urethral lesions can occur, making uretrocystography mandatory for some researchers (3.4).

Many studies in the literature analyze diagnoses and conduct in patients who are victims of gunshot wounds to the genitalia (5.6); however studies with a significant casuistic that solely analyze penile lesions are rare (7). Studies that analyze conduct in urethral gunshot wounds are also rare $(6,7)$.

The objective of the present study is to demonstrate the main aspects in the diagnosis, treatment and follow-up of 43 patients with penile lesions from gunshot wounds.

\section{MATERIALS AND METHODS}

In the period between January 1990 and January 2005, 43 patients with penile lesions from gunshot wounds were retrospectively assessed in our institution. The patient's ages varied between 16 and 54 years (mean age 28 years). The period of time between the trauma and attendance at the hospital varied from 1 to 4 hours (mean of 2 hours and 30 minutes). The location of the lesion, the presence of as- 
sociated lesions, the performance of complementary exams, surgical treatment, postoperative complications and patient follow-up were all analyzed.

The main form of diagnosis assessment was anamnesis and physical examination (Figure-1). In 5 cases $(11.6 \%)$, there was a need for complementary exams. Ultrasound was used in 2 cases to exclude corpus cavernosum lesions, and in 3 cases a cystourethrogram was performed due to a suspicion of urethral lesions.

The surgical technique employed was a subcoronal incision with degloving of the penis and exposure of the corpus cavernosum and urethra.
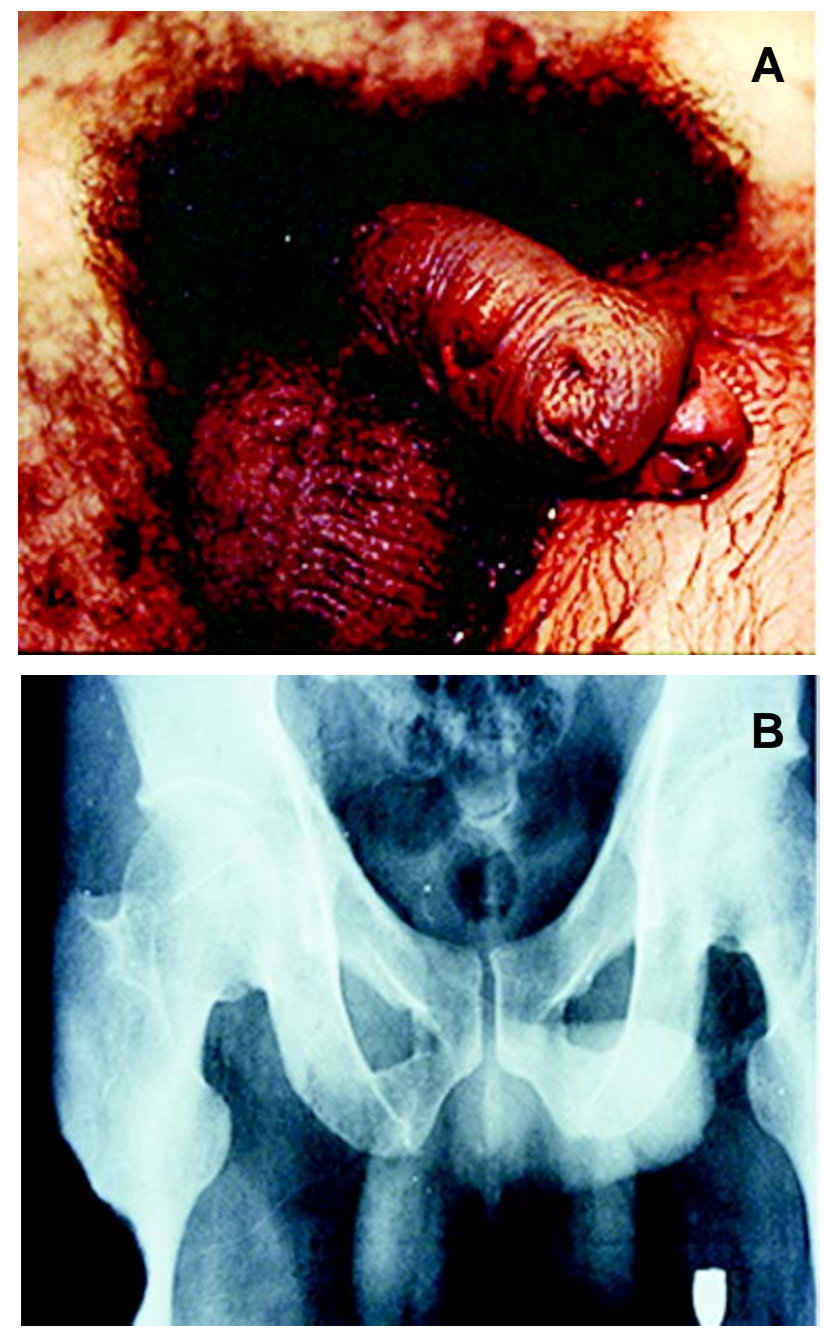

Figure $1-A)$ Transfixing penile lesion associated to distal urethra and glandar lesion. B) X-ray demonstrates the location of the bullet in the external face of the thigh.

\section{RESULTS}

Of the 43 cases analyzed, 41 were submitted to surgical exploration (95.3\%) and only 3 received conservative treatment (4.7\%). Table-1 outlines the lesions found during surgical exploration. In 37 cases we found penile lesions involving the corpus cavernosum; the remaining 4 operated patients did not present lesions either in the corpus cavernosum, urethra or testicles, and presented lesions only in superficial structures. Associations to testicular lesions occurred in 10 cases while association to anterior urethra lesions occurred in 14 cases. Extra-genital lesions were present in 19 patients (44.2\%), with thigh lesions appearing most commonly, as shown in Table2.

Lesions in the corpus cavernosum were treated with local debridement and primary closure with absorbable sutures. Testicular injuries were treated with orchiectomy in 6 cases $(60 \%)$ and reconstruction in 4 cases $(40 \%)$.

Of the 14 cases featuring anterior urethra lesions, $11(78.5 \%)$ had the lesion diagnosed during surgery. Cystostomy and reconstruction of the ure-

Table 1 - The table shows the location of the lesions found in 43 victims of gunshot wounds to the penis.

\begin{tabular}{lcc}
\hline Location of the Lesion & Cases & \% \\
\hline Corpus cavernosum & 13 & 30.2 \\
Testicle + corpus cavernosum & 10 & 23.2 \\
Urethra + corpus cavernosum & 14 & 32.6 \\
Superficial lesion & 6 & 14.0 \\
\hline
\end{tabular}

Table 2 - Location of extragenital lesions found in 43 victims of gunshot wounds to the penis.

\begin{tabular}{lrr}
\hline Location of the Lesion & Cases & \% \\
\hline Thigh & 15 & 34.8 \\
Bone lesion & 6 & 13.9 \\
Vascular lesion & 3 & 6.9 \\
Intra-abdominal lesion & 10 & 23.2 \\
Absence of extragenital lesions & 24 & 55.8 \\
\hline
\end{tabular}


thra were performed later in 2 cases and the other 12 cases received immediate treatment of the lesions. In 4 patients with extended partial lesions $(>2 \mathrm{~cm}$ ) of the pendular urethra, a primary suture over a urethral catheter was performed; 3 of these patients (75\%) required a second procedure due to postoperative urethral stenosis, while 1 patient (25\%) developed urethral cutaneous fistula which was treated with prolonged urethral catheterization.

In 3 patients featuring pendular urethra lesions ( 1 with a total lesion and 2 with partial lesions), a cutaneous urethrostomy was performed and a later reconstruction was conducted by rotating the cutaneous flap; there was no postoperative stenosis or fistulas in any of the 3 cases. In 5 patients, the lesion was to the bulbar urethra (distal portion). In 1 case a primary suture was performed over the catheter and in 4 cases termino-terminal anastomosis was performed, presenting no postoperative complications. In the 2 patients where the cystostomy was performed as a primary treatment, late stenosis occurred, requiring urethral reconstruction.

A greater than 6 month follow-up was possible in 30 patients -11 with urethral lesions, 10 with lesions only in the corpus cavernosum and 9 with testicle and corpus cavernosum lesions. None of the 30 cases presented penile curvature or erectile dysfunction determining significant impact on the quality of sexual activity.

\section{COMMENTS}

Success in the treatment of penile lesions from gunshot wounds depends on early surgical exploration, debridement of the wounded structures and primary lesion repair (8-10). Conservative treatment in these cases is controversial, except when clinical evaluation strongly indicates the presence of only superficial lesions (1). In our study, there was no surgical exploration in only $4.8 \%$ of the cases and clinical indications in those cases were only of superficial lesions. During physical examination, these patients presented presence of a minimum hematoma that allowed an adequate palpation of corpora cavernosa and testicles, with absence of lesions con- firmed by ultrasound. In the other 4 cases $(9.5 \%)$, the patients were submitted to surgical exploration based on the findings of physical examination; however, only lesions in superficial structures were found in these cases.

The use of the injury severity score from the American Association for the Surgery of Trauma (AAST) is being utilized to facilitate uniform treatment of genitalia lesions $(1,11)$. According to this classification, superficial lesions and contusions are classified as degree I and can be conservatively treated. Lesions classified as degree II (Buck fascia lacerations without tissue loss), degree III (cutaneous avulsion or laceration through glands and meatus, or urethral or cavernosum lesions less than $2 \mathrm{~cm}$ in area), degree IV (partial penectomy or urethral or cavernosum lesions more than $2 \mathrm{~cm}$ in area), and degree $\mathrm{V}$ (total penectomy) shall be treated with surgery $(1,11)$.

The majority of patients with penetrating lesions in the genitalia should be submitted to immediate surgical intervention; however in doubtful cases of degree I lesions according to AAST, the use of ultrasound to exclude the lesions of the corpus cavernosum can be useful even though it is an operator-dependent examination (12-15). In our sample, both patients that were not submitted to surgical intervention were submitted to ultrasound, which only confirmed the presence of superficial lesions.

The adequate exploration and reconstruction of corpora cavernosa seem to prevent the late development of complications, such as erectile dysfunction and penile curvature. This observation was support in the wide experience with primary reconstructions in the case of penile contusion traumatisms (15).

According to the literature, lesions of the urethra vary from 17 to $22 \%$ of the cases of penile lesions from gunshot wounds $(1,10)$. In our sample, we found lesions of the urethra in 14 patients (33\%). Due to this important percentage, and since this is a low cost, easily performed and highly precise exam, many authors recommend the performance of routine cystourethrogram $(1,4,16)$ independently of a doubtful clinical exam (presence of blood in the meatus, urinary retention, prostatic elevation upon rectal touch). In our study, we performed a cystourethrogram in only 
3 cases where the exam confirmed the lesion, and in 11 cases the lesion was identified only during surgical intervention.

In cases of suspicion of urethral lesion at initial admission, retrograde cystourethrogram should be performed as the patient's clinical conditions allow. Penile degloving for surgical exploration should always be performed after urethral catheterization, as this maneuver will help identify transoperatively the urethral lesions.

In the cases of urethral lesions, primary repair is the option of choice (6), except in cases where there is an extended loss of urethral tissue (4), when staged treatment is the best choice. Of the 14 patients with urethral lesions, we performed cystostomy and late urethral reconstruction in only 2 cases (14.2\%). Lesions of the pendular urethra (especially in distal and glandar portions) should be treated in a staged form due to the high frequency of complications.

Performing an urethrostomy as an initial procedure seems to be an interesting choice since it will allow reconstruction using tissues where the thermal late lesion of the bullet is already defined. Terminoterminal anastomosis is possible for bulbar urethral lesions limited to the penoscrotal junction when there is an extension less than $1.5 \mathrm{~cm}$. When the urethral lesion is only partial (less than $50 \%$ of the urethral circumference with longitudinal extension less than $1 \mathrm{~cm}$ ), we believe that the primary closure of the lesion over a catheter can be done; otherwise the option of a termino-terminal anastomosis or staged treatment should be done, depending on the location of the lesion.

In the absence of a trained urological medical team to reconstruct the lesion, or when the lesion is extended to the more distal portions of the bulbar urethra or to the posterior urethra, or when the clinical conditions do not allow exploration of the lesion, cystostomy shall be performed as a primary treatment.

\section{CONCLUSIONS}

Penile lesions from gunshot wounds shall be treated through an immediate surgical intervention, except in cases where there are only superficial le- sions that should be confirmed by ultrasound. The primary repair of corpus cavernosum lesions seems to prevent the late development of complications. Urethral lesions should be treated, whenever possible, at the moment of surgical intervention, while cases of extended lesions or those localized in distal portions of the urethra should be submitted to 2step surgery.

\section{CONFLICT OF INTEREST}

None declared.

\section{REFERENCES}

1. Mohr AM, Pham AM, Lavery RF, Sifri Z, Bargman V, Livingston DH: Management of trauma to the male external genitalia: the usefulness of American association for the surgery of trauma organ injury scales. J Urol. 2003; 170: 2311-2315.

2. Morey AF, Metro MJ, Carney KJ, Miller KS, McAninch JW: Consensus on genitourinary Trauma: external genitalia. BJU Int. 2004; 94: 507-15.

3. van der Horst C, Martinez Portillo FJ, Seif C, Groth W, Junemann KP: Male genital injury: diagnostics and treatment. BJU Int. 2004; 93: 927-30.

4. Tiguert R, Harb JF, Hurley PM, Gomes De Oliveira J, Castillo-Frontera RJ, Triest JA, et al.: Management of shotgun injuries to the pelvis and lower genitourinary system. Urology. 2000; 55: 193-7.

5. Gomez RG, Castanheira AC, McAninch JW: Gunshot wounds to the male external genitalia. J Urol. 1993; 150: 1147-9.

6. Husmann DA, Boone TB, Wilson WT: Management of low velocity gunshot wounds to the anterior urethra: the role of primary repair versus urinary diversion alone. J Urol. 1993; 150: 70-2.

7. Mydlo JH, Harris CF, Brown JG: Blunt, penetrating and ischemic injuries to the penis. J Urol. 2002; 168: 1433-5.

8. Brandes SB, Buckman RF, Chelsky MJ, Hanno PM: External genitalia gunshot wounds: a ten-year experience with fifty-six cases. J Trauma. 1995; 39: 266-71; discussion 271-2.

9. Monga M, Moreno T, Hellstrom WJ: Gunshot wounds to the male genitalia. J Trauma. 1995; 38: 855-8. 
10. Cline KJ, Mata JA, Venable DD, Eastham JA: Penetrating trauma to the male external genitalia. J Trauma. 1998; 44: 492-4.

11. Moore EE, Malangoni MA, Cogbill TH, Peterson NE, Champion HR, Jurkovich GJ, et al.: Organ injury scaling VII: cervical vascular, peripheral vascular, adrenal, penis, testis, and scrotum. J Trauma. 1996; 41: 523-4.

12. Miller S, McAninch JW: Penile fracture and soft tissue injury. In: Traumatic and Reconstructive Urology. Edited by JW. McAninch. Philadelphia, WB. Saunders. 1996; chapt. 59, pp. 693-8.
13. Dierks PR, Hawkins H: Sonography and penile trauma. J Ultrasound Med. 1983; 2: 417-9.

14. Martinez Perez E, Arnaiz Esteban F, Perez Arbej JA, Nogueras Gimeno MA, Crespo Mayor V, Espuela Orgaz R: Fracture of the penis: two new cases. Review of the literature. Usefulness of echography. Arch Esp Urol. 1997; 50: 1099-102.

15. Koifman L, Cavalcante AG, Manes CH, Filho DR, Favorito LA: Penile fracture - Experience in 56 cases. Int Braz J Urol. 2002; 29: 35-39.

16. Hall SJ, Wagner JR, Edelstein RA, Carpinito GA: Management of gunshot injuries to the penis and anterior urethra. J Trauma, 1995; 38: 439-43.

Accepted after revision:

September 30, 2005

\author{
Correspondence Address: \\ Dr. Luciano Alves Favorito \\ Rua Professor Gabizo 104/201 \\ Rio de Janeiro, RJ, 20271-320, Brazil \\ Fax: + $55213872-8802$ \\ E-mail: favorito@uerj.br
}

\title{
EDITORIAL COMMENT
}

Gunshot lesions of penis are often mutilating with a high incidence of aesthetic, physical, dysfunctional and psychological post-trauma morbidity and urinary and sexual disability. Most of patients develop significant mental disorders after trauma in genitalia area and for this reason, when you deal with this matter, it is mandatory to maintain penis function and cosmesis.

In this issue of the International Braz J Urol, Cavalcanti and colleagues retrospectively reviewed 43 patients with a penile lesion by gunshot. Penile lesions involved the corpus cavernosum in 37 cases and in 14 cases, they found a urethral lesion. Authors stated that the primary repair of corpus cavernosum lesions is the accepted method of treatment in patients who have lesions involving the corpora cavernosa, but not urethra, and this surgical strategy may prevent the late development of complications, such as penile curvature as well as erectile dysfunction.

Urethral lesion treatment, however, represent the main challenge for urologists, who have to face with acute trauma of penis involving urethra. We do agree with Cavalcanti et al., that urethral lesions should be treated, whenever possible, at the moment of early surgical exploration but cases of extended lesions or those localized in distal portions of the urethra with significant loss of tissue should be submitted to 2-step surgery.

Penile urethroplasty, whether a one stage or a multistage repair, performed after a gunshot trauma of penis involving urethra, is intrinsically prone to 
complications such as hematoma or infection, which in turn can lead to secondary complications such as fistula, cosmetic defects and stenosis. Penile urethral stricture always represents a more difficult challenge in patients with failed primary repair after a trauma. Generally, it is the consequence of the corpus spongiosum damage (lack of it or fibrosis) because it represents the basic tissue to provide the adequate vascular and mechanical support to urethra. Authors did not report exactly what specific technique of urethroplasty was used in their series or what material they used as transplanted substitute material. In 3 patients with pendular urethra lesions ( 1 with a total lesion and 2 with partial lesions), a cutaneous urethrostomy was performed and a later reconstruction was conducted by rotating the cutaneous flap. We would like to advise that after a gunshot lesion of penis you can have a defect of penis and preputial skin and it will become scarred after the first surgical intervention. Therefore, we suggest avoiding the use of skin, and we prefer buccal mucosa graft when one chooses a multistage procedure.

All patients who underwent urethral repair after a gunshot trauma should be fully informed that any surgical strategy might be complicated by stenosis and that more than two surgical steps could be necessary to restore both urethral potency and acceptable aesthetic appearance of genitalia. The best strategy to repair urethra after the gunshot trauma remains unknown, however, it is probably the one with which the surgeon is most comfortable and it could be appropriately tailored on the single patient. No single technique is appropriate for all the complex situations after a penis trauma. It is a useful reminder to readers that this is a challenging surgery and should not therefore be undertaken by surgeons who only dabble in this field. In the near future, tissue-engineering technology could provide a new urethra with normal spongiosum tissue around the mucosal-urothelium strip, offering urologists a higher quality substitute material with which to work in selected situation.

Finally, I congratulate the authors in presenting this informative series and a very candid account of their experience with gunshot lesions of penis. I think that the reader would fully concur with my take home message: urethral lesion by a gunshot trauma can be challenging and difficult to correct, even in the hands of experienced urethral surgeons.

\author{
Dr. Massimo Lazzeri \\ Department of Urology \\ Casa di Cura Santa Chiara \\ Firenze, Italy \\ E-mail:lazzeri.m@tiscali.it
}

\section{EDITORIAL COMMENT}

Gunshot wounds of the penis can be devastating injuries resulting in soft tissue loss, severe cosmetic deformities, erectile dysfunction and urethral injury. Any one of these results can cause tremendous problems for the patient and they involve urological surgery.

Virtually all of these injuries come from handguns, which generally are of low velocity, and result in less soft tissue destruction. Penile tissue is very vascular and debridement should be very conservative in order to preserve maximal amount of soft tissue. Lacerations of the tunica albuginea should be closed with absorbable suture without an attempt to explore or debride the cavernous erectile tissue. In most cases, this preserves erectile function, as shown by Cavalcanti and his associates. Operative explora- 
tion should be planned in all patients unless careful examination can demonstrate only superficial skin injury without any large defect.

I believe that all patients with penile gunshot injuries should have preoperative retrograde urethrography. Certainly, most will be detected during operative exploration, as demonstrated by Cavalcanti and his colleagues. However, retrograde urethrography can accurately diagnose the injury preoperatively and primary reconstruction can be planned with initial surgical exploration. Without urethrography, a missed urethral injury could result in major complications for the patient. This was not true in Calvalcanti's series, which indicate the careful evaluation of the urethra during surgical exploration. In most situations, the urethra can be repaired at the initial surgery as shown in this series.

Remarkably, these patients usually do extremely well if managed as shown in this paper. Erectile function and cosmetic appearance are generally preserved and urethral strictures seldom occur when proper repair has been done.

This large series of penile gunshot wounds provides excellent guidelines for diagnosis and management, and demonstrates the excellent outcomes, which can be achieved.

Dr. Jack W. McAninch

Department of Urology, University of California

Chair of Urology, San Francisco General Hospital

San Francisco, California, USA

E-mail: jwm@itsa.ucsf.edu

\section{EDITORIAL COMMENT}

Large series describing genital gunshot wounds are rare in the literature. In the past decade, there are fewer than 6 published reports on the subject, describing only about 40 patients. This Brazilian report comes from an area of Rio de Janeiro with a vast and largely untapped experience with the treatment of genitourinary injuries from gunshot.

Need for urethrogram - In this series, urethrography was only rarely $(7 \%)$ used. Caution must be exercised as $33 \%$ of patients ultimately had urethral injury. In the hands of these experienced genitourinary trauma surgeons, the urethral lesions were detected and repaired, but in centers with less experience, it is probably prudent to use preoperative urethrography more liberally than seen in this report. The authors also always placed a urethral catheter before exploration, and you should too.
Ultrasound - Adjuvant studies to determine if penile gunshot wounds injured the corpora cavernosa were used rarely in this report (5\%), but this is the first such description of this protocol that I have seen. In general, it is probably a good idea to avoid exploration in those penile gunshot wounds that clearly do not breach the corpora, by either exam or imaging examination. In this series, $10 \%$ of patients had negative exploration, but I would suggest that $10 \%$ negative explorations is preferable to missing corporal or (especially) urethral injuries.

How to repair pendulous urethral injuries? A source of continued discussion in the literature is whether or not primary repair is preferable to merely diverting the urine with suprapubic tube or urethral catheter. In this series, repair was opted for in a majority of cases, but only some did well. In those 
with pendulous urethral injury, all the 4 primarily repaired patients did poorly (developing fistula or stricture) while 3 were treated in a staged fashion, as in a Johanson urethroplasty. Like the authors, we tend to primarily repair those injuries with limited tissue damage, although in our experience complications were less common, perhaps because of lower caliber gunshot wounds or other patient factors. We also do not hesitate to exteriorize the injured urethral segment and return for definitive repair at a later date, as the authors did, especially in more severe injuries.

How to repair bulbar urethral injuries? All of the 5 repaired bulbar urethral injuries did well, and a $4 / 5$ of these required anastomotic repair after excision of the injured segment. This technique is hardly reported in the literature previously, and should be considered by all those faced with this injury.

Dr. Richard A. Santucci

Chief of Urology, Detroit Receiving Hospital Associate Professor, Wayne State University

Detroit, Michigan, USA

E-mail:rsantucc@med.wayne.edu 